\title{
Protocatechuic Acid Inhibits Rat Pheochromocytoma Cell Damage Induced by a Dopaminergic Neurotoxin
}

\author{
Hong-Ning Zhang, ${ }^{a, b}$ Chun-Na An, ${ }^{a, b}$ Man Xu,${ }^{c}$ De-An Guo, ${ }^{c}$ Min LI, ${ }^{a}$ and Xiao-Ping Pu ${ }^{*, a, b}$ \\ ${ }^{a}$ State Key Laboratory of Natural and Biomimetic Drugs, Peking University; ${ }^{b}$ Department of Molecular and Cellular \\ Pharmacology, Peking University; and ${ }^{c}$ Department of Natural Drugs, School of Pharmaceutical Science, Peking \\ University; 38 Xueyuan Road, Haidian District, Beijing 100191, P. R. China.
}

Received June 23, 2009; accepted August 10, 2009; published online August 14, 2009

\begin{abstract}
Parkinson's disease (PD) is characterized by the progressive degeneration of dopaminergic neurons in the substantia nigra (SN) with the presence of $\alpha$-synuclein inclusions termed Lewy bodies. The aggregation of $\alpha$ synuclein into oligomeric species affects neuronal viability, having a causal role in the development of PD. The neuroprotective effects of protocatechuic acid (PAc) have been reported. However, the effects of PAc on tyrosine hydroxylase (TH) and $\alpha$-synuclein in rat pheochromocytoma (PC12) cells treated with 1-methyl-4-phenylpyridinium ion $\left(\mathrm{MPP}^{+}\right)$remains unclear. In this study, we demonstrated that PAc inhibited the cytotoxicity, apoptotic morphology, reduction of TH expression and abnormal oligomeration of $\alpha$-synuclein in PC12 cells treated with $\mathrm{MPP}^{+}$. Taken together, our results indicate that the neuroprotective effects of PAc on PC12 cells treated with $\mathrm{MPP}^{+}$is related to the inhibition of the oligomerization of $\alpha$-synuclein.
\end{abstract}

Key words protocatechuic acid; neuroprotection; rat pheochromocytoma cell; 1 -methyl-4-phenylpyridinium ion; $\alpha$-synuclein

Parkinson's disease (PD) is the second most common neurodegenerative disorder worldwide, and is characterized by the progressive loss of dopaminergic neurons in the substantia nigra $(\mathrm{SN}){ }^{1-3)}$ The clinical symptoms of PD mainly include rest tremors, rigidity, bradykinesia and postural abnormalities. ${ }^{4,5)}$ At present, a number of genetic factors such as Parkin, $\alpha$-synuclein, DJ-1, PINK1 and LRRK2, as well as environmental factors such as exposure to pesticides, neurotoxins and heavy metals, have been considered to contribute to the onset of PD.,7) 1-Methyl-4-phenyl-1,2,3,6-tetrahydropyridine (MPTP), one of the selective nigrostriatal dopaminergic neurotoxins, was the first conclusive demonstration of a link between environmental toxin exposure and the development of PD. ${ }^{8,9)}$ The toxicity of MPTP depends on its pyridinium derivative, 1-methyl-4-phenylpyridinium ion $\left(\mathrm{MPP}^{+}\right) \cdot{ }^{10)}$ One of the hallmarks of PD is the presence of $\alpha$ synuclein inclusions, termed Lewy bodies, in the dopaminergic neurons of $\mathrm{SN}$ and other brain regions such as cortex and basal forebrain nuclei. $\alpha$-Synuclein is generally thought to play an important role in synaptic plasticity and to function under normal expression, but it has neuropathological effects when overexpressed or mutated and induces the formation of Lewy bodies. ${ }^{11,12)}$

Protocatechuic acid (PAc) (Fig. 1) is a water-soluble monomeric phenolic acid with strong free radical scavenging effects. ${ }^{13)}$ The neuroprotective effects of PAc have previously been reported. ${ }^{14)}$ However, little is known about the effects of PAc on tyrosine hydroxylase (TH) and oligomerization of $\alpha$ synuclein in rat pheochromocytoma (PC12) cells treated with $\mathrm{MPP}^{+}$. The aim of this study was to investigate the effects of PAc on these aspects.

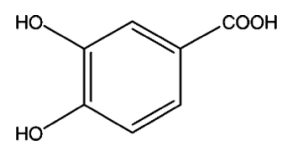

Fig. 1. The Structure of PAc

\section{MATERIALS AND METHODS}

Materials PAc (purity $>99 \%$ ) was purchased from the National Institute for the Control of Pharmaceutical and Biological Products (China). F12K cell culture medium was purchased from Invitrogen (Carlsbad, CA, U.S.A.). Donor equine serum was purchased from Hyclone (Logan, UT, U.S.A.) and fetal bovine serum from Si-ji-qing (Hangzhou, China). MPP $^{+}$, 3-(4,5-dimethylthiozol-2-yl)-2,5-diphenyltetrazolium bromide (MTT) and hoechst 33342 were purchased from Sigma (St. Louis, MO, U.S.A.). Rabbit TH monoclonal antibody was purchased from Chemicon (Temecula, CA, U.S.A.). The bicinchoninic acid (BCA) protein assay kit was purchased from Perice (Rockford, IL, U.S.A.). Mouse $\alpha$ synuclein monoclonal antibody and rabbit $\beta$-actin polyclonal antibody were purchased from Santa Cruz (Santa Cruz, CA, U.S.A.). Fluoresceine isothiocyanate (FITC)-conjugated goat anti-rabbit immunoglobin $\mathrm{G}(\mathrm{IgG})$ and horseradish peroxidase (HRP)-conjugated goat anti-mouse IgG were purchased from Zhong-shan Goldenbridge (Beijing, China). HRP-conjugated goat anti-rabbit IgG was purchased from Ding-guo (Beijing, China).

Cell Culture The PC12 cell line was purchased from the Shanghai Cell Bank, Chinese Academy of Sciences and grown in F12K medium with glutamine supplemented with $15 \%$ donor equine serum and $2.5 \%$ fetal bovine serum at $37^{\circ} \mathrm{C}$ in a humidified $5 \% \mathrm{CO}_{2}$ incubator. All experiments were performed at a cell density of $4 \times 10^{4} / \mathrm{cm}^{2}$.

Cell Viability Analysis Cells were pre-treated with PAc $(0.33 \mathrm{~mm}, 0.65 \mathrm{~mm}, 1.30 \mathrm{~mm})$ for $6 \mathrm{~h}$, then $1 \mathrm{~mm} \mathrm{MPP}{ }^{+}$was added and the incubation continued for $24 \mathrm{~h}$. After treatment, MTT solution was added and the cells were further incubated for $4 \mathrm{~h}$. The formazan crystals were dissolved with sodium dodecyl sulfate (SDS) solution and measured with a microplate reader. Cell viability was expressed as a percentage of the control cells.

Transmission Electron Microscopy (TEM) Cells were pre-treated with $1.30 \mathrm{~mm}$ PAc for $6 \mathrm{~h}$, then $1 \mathrm{~mm} \mathrm{MPP}{ }^{+}$was added and the incubation continued for $24 \mathrm{~h}$. After treatment, 
cell precipitates were fixed with glutaraldehyde, post-fixed with osmium tetroxide, dehydrated through a graded alcohol series, soaked in epoxy resin and acetone and embedded into epoxy resin. Ultra-thin sections were then cut and stained with uranyl acetate and lead citrate and examined under a JEM-100 transmission electron microscope (Jeol, Japan).

TH Immunofluorescence Cells were pre-treated with PAc $(0.33 \mathrm{~mm}, 0.65 \mathrm{~mm}, 1.30 \mathrm{~mm})$ for $6 \mathrm{~h}$, then $1 \mathrm{~mm} \mathrm{MPP}$ was added and the incubation continued for $24 \mathrm{~h}$. After treatment, cells were fixed with 4\% paraformaldehyde solution for $30 \mathrm{~min}$, penetrated with $0.3 \%$ Triton X-100 solution for $5 \mathrm{~min}$, blocked with $10 \%$ goat serum solution for $30 \mathrm{~min}$, incubated with rabbit monoclonal $\mathrm{TH}$ antibody $(1: 300)$ overnight, immuno-reacted with FITC-conjugated goat antirabbit secondary antibody for $1 \mathrm{~h}$, stained with hoechst 33342 for $10 \mathrm{~min}$ and visualized under a TCS spII confocal laser-scanning microscope (CLSM) (Leica, Germany).

$\alpha$-Synuclein Western Blot Cells were pre-treated with PAc $(0.33 \mathrm{~mm}, 0.65 \mathrm{~mm}, 1.30 \mathrm{~mm})$ for $6 \mathrm{~h}$, then $1 \mathrm{~mm} \mathrm{MPP}$ was added and the incubation continued for $24 \mathrm{~h}$. After treatment, cell precipitates were lysed and centrifuged. Supernatants were quantified and run on a $12.5 \%$ sodium dodecyl sulfate (SDS) polyacrylamide gel. The separated proteins were blotted onto a poly(vinylidene fluoride) (PVDF) membrane and processed for immunodetection. An $\alpha$-synuclein monoclonal antibody was used as the primary antibody $(1: 1000)$ and HRP-conjugated IgG as the secondary antibody. $\beta$-Actin was used as an internal standard. An enhanced chemiluminescence detection system was applied to detect the target proteins. The results of Western blot were analyzed and quantified by Quantity One software (Bio-Rad, U.S.A.).

Statistical Analysis Data are expressed as mean \pm S.D. of three independent experiments. Statistical analysis was performed using Student's $t$-test and one-way analysis of variance (one-way ANOVA) by SPSS 13.0 software and differences were considered to be statistically significant when $p<0.05$.

\section{RESULTS}

PAc Inhibited the Cytotoxicity in PC12 Cells Treated with MPP $^{+}$We first assessed whether PAc can inhibit the cytotoxicity of cells exposed to $\mathrm{MPP}^{+}$by MTT assay. As shown in Fig. 2, PAc significantly inhibited the cytotoxicity induced by $\mathrm{MPP}^{+}$in a dose-dependent manner. $\mathrm{MPP}^{+}$treatment resulted in $28.9 \%$ cell death. However, pre-treatment with PAc reduced the $\mathrm{MPP}^{+}$-induced cell death to $20.7 \%$, $8.8 \%$ and $0 \%$, at concentrations of $0.33 \mathrm{~mm}, 0.65 \mathrm{~mm}$ and $1.30 \mathrm{~mm}$, respectively $(p<0.05)$. The cell viability of PC12 cells treated with PAc alone showed no changes.

PAc Ameliorated the Apoptotic Morphology in PC12 Cells Treated with $\mathrm{MPP}^{+}$The protective effects of PAc on $\mathrm{MPP}^{+}$-treated cells were investigated by TEM. MPP ${ }^{+}$treatment resulted in apoptosis of cells, characterized by chromatin condensation and fragmentation and mitochondria engorgement. Cells pre-treated with PAc (1.30 mM) before $\mathrm{MPP}^{+}$treatment, however, exhibited a relatively normal cellular morphology characterized by chromatin dispersion and normal mitochondria (Fig. 3).

PAc Inhibited the Reduction of TH Expression in PC12 Cells Treated with MPP $^{+}$The dopaminergic neuron phe-

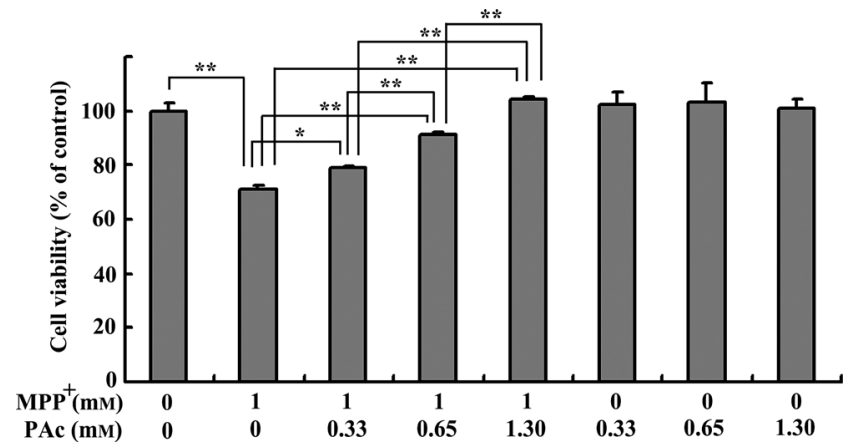

Fig. 2. Pre-treatment with PAc $(0.33 \mathrm{~mm}, 0.65 \mathrm{~mm}, 1.30 \mathrm{~mm})$ for $6 \mathrm{~h}$ Inhibited the Cytotoxicity in PC12 Cells Treated with $\mathrm{MPP}^{+}(1 \mathrm{~mm})$ for $24 \mathrm{~h}$ by MTT Assay

The cell viability of PC12 cells treated with PAc alone showed no changes. Data are mean \pm S.D. of three independent experiments. $* p<0.05, * * p<0.01$.
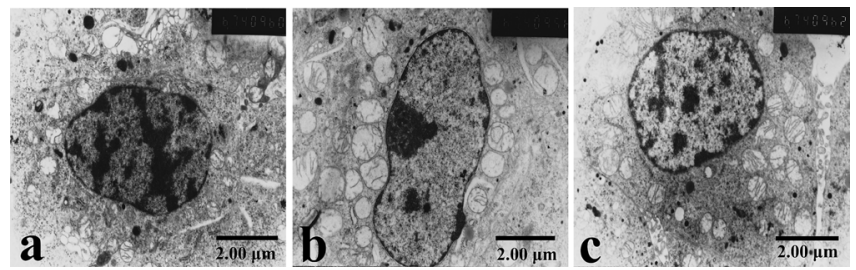

Fig. 3. Pre-treatment with PAc $(1.30 \mathrm{~mm})$ for $6 \mathrm{~h}$ Inhibited the Apoptosis in PC12 Cells Treated with $\mathrm{MPP}^{+}(1 \mathrm{mM})$ for $24 \mathrm{~h}$ by TEM

(a) Control; (b) $1 \mathrm{~mm} \mathrm{MPP}{ }^{+}$; (c) $1.30 \mathrm{~mm} \mathrm{PAc}+1 \mathrm{~mm} \mathrm{MPP}^{+}$. Magnification: $6740 \times$ Scale bar $=2.00 \mu \mathrm{m}$. Data are representative of three independent experiments.
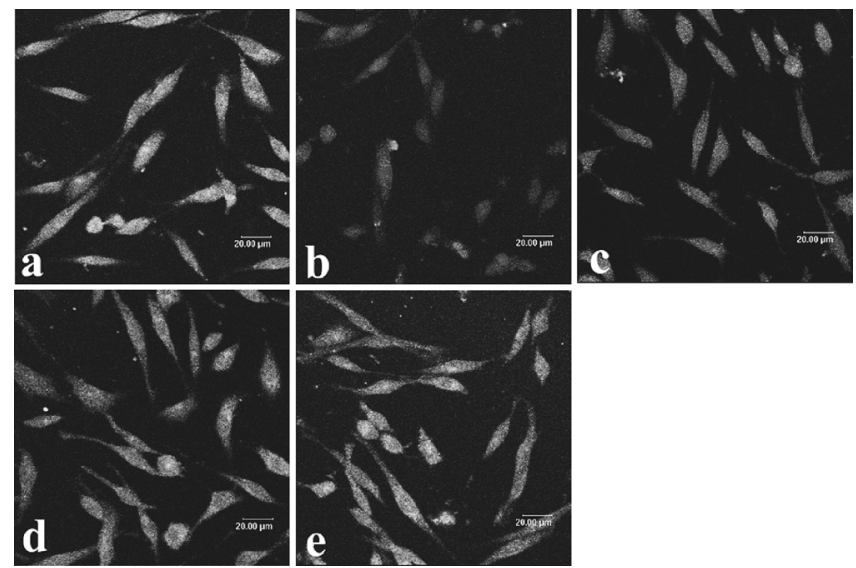

Fig. 4. Pre-treatment with PAc $(0.33 \mathrm{~mm}, 0.65 \mathrm{~mm}, 1.30 \mathrm{~mm})$ for $6 \mathrm{~h}$ Inhibited the Reduction of TH Expression in PC12 Cells Treated with MPP (1 $\mathrm{mm}$ ) for $24 \mathrm{~h}$ under CLSM

Green fluorescence represents the expression of $\mathrm{TH}$ and blue fluorescence represents the location of nuclei. (a) Control; (b) $1 \mathrm{~mm} \mathrm{MPP}{ }^{+}$; (c) $0.33 \mathrm{~mm} \mathrm{PAc}+1 \mathrm{~mm} \mathrm{MPP}^{+}$; (d) $0.65 \mathrm{~mm} \mathrm{PAc}+1 \mathrm{~mm} \mathrm{MPP}+; 1.30 \mathrm{~mm} \mathrm{PAc}+1 \mathrm{~mm} \mathrm{MPP}^{+}$. Scale bar $=20.00 \mu \mathrm{m}$. Data are representative of three independent experiments.

notype of PC12 cells was identified by examining the expression of $\mathrm{TH}$ using immunofluorescence. $\mathrm{MPP}^{+}$treatment resulted in neurites decurtation and decreased fluorescence intensity for TH of cells. However, cells pre-treated with PAc $(0.33 \mathrm{~mm}, 0.65 \mathrm{~mm}, 1.30 \mathrm{~mm})$ before $\mathrm{MPP}^{+}$treatment, displayed elongated neurites and increased fluorescence intensity for TH. Green fluorescence represents the expression of $\mathrm{TH}$ and blue fluorescence represents the location of nuclei (Fig. 4).

PAc Inhibited the Oligomerization of $\alpha$-Synuclein in 

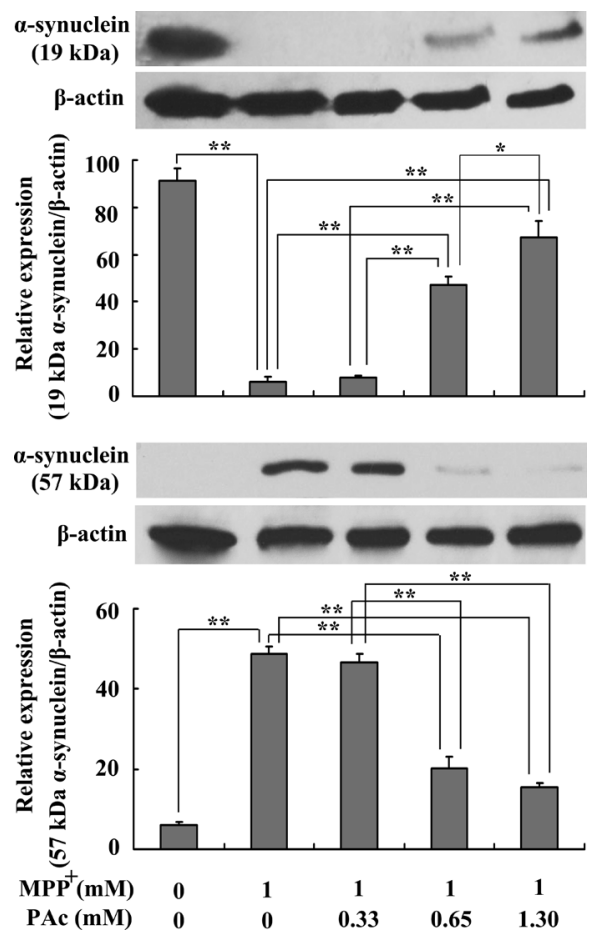

Fig. 5. Pre-treatment with PAc $(0.65 \mathrm{~mm}, 1.30 \mathrm{~mm})$ for $6 \mathrm{~h}$ Inhibited the Oligomerization of $\alpha$-Synuclein in PC12 Cells Treated with $\mathrm{MPP}^{+}(1 \mathrm{~mm})$ for $24 \mathrm{~h}$ by Western Blot Analysis

$\beta$-Actin was used as a internal standard. Data are mean \pm S.D. of three independent experiments. $* p<0.05, * * p<0.01$.

PC12 Cells Treated with MPP $^{+} \alpha$-Synuclein was detected by Western blot analysis. The expression of monomeric $(19 \mathrm{kDa})$ or oligomeric $(57 \mathrm{kDa}) \alpha$-synuclein decreased or increased in $\mathrm{MPP}^{+}$-treated cells, respectively. However, PAc $(0.65 \mathrm{~mm}, 1.30 \mathrm{~mm})$ significantly reduced the abnormal decrease or increase of monomeric or oligomeric $\alpha$-synuclein in cells treated with $\mathrm{MPP}^{+}$(Fig. 5).

\section{DISCUSSION}

In the present study, we have confirmed that PAc has neuroprotective effects on $\mathrm{PC} 12$ cells treated with $\mathrm{MPP}^{+}$by MTT assay, TEM morphology and TH immunofluorescence.

Considerable evidences have shown that dopaminergic neurons of the SN in PD patients show characteristics of apoptosis, including nuclear condensation, chromatin fragmentation, and formation of apoptotic-like bodies. ${ }^{1,2)} \mathrm{MPP}^{+}$ has also been found to cause apoptosis in dopaminergic PC12 cells. ${ }^{15)}$ TEM results indicated that PAc inhibits the chromatin condensation and margination and mitochondria engorgement, typical features of apoptosis, in PC12 cells treated with $\mathrm{MPP}^{+}$.

Dopamine (DA) is a main neurotransmitter of dopaminergic neurons in SN-striatum and it is synthesized from tyrosine by the catalysis of $\mathrm{TH}$, the rate-limiting enzyme of DA production process. Naturally, the expression of $\mathrm{TH}$ in $\mathrm{SN}$ becomes a parameter reflecting the activity of dopaminergic neurons. It has reported that the dopaminergic neuron phenotype of PC12 cells was identified by examining the expression of TH using immunofluorescence. ${ }^{16)}$ Our results demonstrated that PAc can inhibit the reduction of $\mathrm{TH}$ expression in
PC12 cells induced by $\mathrm{MPP}^{+}$.

To reveal the possible mechanism by which PAc inhibits the neurotoxicity in PC12 cells induced by $\mathrm{MPP}^{+}, \alpha$-synuclein was detected by Western blot analysis. The $\alpha$-synuclein aggregation into oligomers, fibrils, up to Lewy bodies affects neuronal viability, having a causal role in the development of $\mathrm{PD}$, and the prevention of these abnormal changes has become the focus of new PD therapeutic efforts. ${ }^{11,12)}$ According to these literatures, when over expressed or mutated by outer stimuluses such as MPTP/MPP ${ }^{+}$, the monomeric $\alpha$ synuclein is easily marched oligomerization forming into oligomers $(57 \mathrm{kDa}) . \mathrm{Xu}$ and colleagues ${ }^{17)}$ have reported that $\mathrm{MPP}^{+}$can increase the expression of oligomeric $\alpha$-synuclein $(57 \mathrm{kDa})$ in PC12 cells. Our Western blot assays showed that PAc significantly inhibits the reduction of monomeric $\alpha$ synuclein $(19 \mathrm{kDa})$ expression and the augmentation of oligomeric $\alpha$-synuclein $(57 \mathrm{kDa})$ expression in $\mathrm{MPP}^{+}$-treated PC12 cells. For these reasons mentioned above, we deduce that PAc inhibits the abnormal oligomerization of $\alpha$-synuclein in PC12 cells induced by $\mathrm{MPP}^{+}$probably through inhibiting the over expression or mutation and stabilizing the physiological level of $\alpha$-synuclein. It has been reported that the inhibition of TH in dopaminergic cells are considered to be tightly linked with dopaminergic cell death caused by the abnormal changes in $\alpha$-synuclein. ${ }^{18,19)}$ In our experiments, we detected that PAc inhibits the reduction of TH expression in $\mathrm{MPP}^{+}$-treated PC12 cells. Therefore, the inhibitory effects of PAc on damages in PC12 cells treated with $\mathrm{MPP}^{+}$detected by MTT assay, TEM morphology and TH immunofluorescene are possibly related to the suppression of the abnormal oligomerization of $\alpha$-synuclein. The significant inhibitory effect of PAc on the oligomerization of $\alpha$-synuclein indicates that this could be one of the main mechanisms for neuroprotective effects of PAc on $\mathrm{MPP}^{+}$-induced toxicity in PC12 cells. Our next research will focus on clarifying the precise mechanisms that the neuroprotective effects of PAc on $\mathrm{PC} 12$ cells induced by $\mathrm{MPP}^{+}$are related to the inhibition of the oligomerization of $\alpha$-synuclein.

In summary, the results of the present study indicate that PAc can protect cultured PC12 cells against damage induced by $\mathrm{MPP}^{+}$. We demonstrate, for the first time, that the neuroprotective effects of PAc, at least in part, are related to the suppression of the oligomerization of $\alpha$-synuclein.

Acknowledgements This work was supported by the National Program for Key Basic Research Projects of China (2004CB518902).

\section{REFERENCES}

1) Lev N., Melamed E., Offen D., Prog. Neuropsychopharmacol. Biol. Psychiatry, 27, 245-250 (2003).

2) von Bohlen und Halbach O., Schober A., Krieglstein K., Prog. Neurobiol., 73, 151-177 (2004).

3) Geng X. C., Song L. W., Pu X. P., Tu P. F., Biol. Pharm. Bull., 27, 797-801 (2004).

4) Singha S., Dikshit M., Brain Res. Rev., 54, 233-250 (2007).

5) Toulouse A., Sullivan A. M., Prog. Neurobiol., 85, 376-392 (2008).

6) Yang Y. X., Wood N. W., Latchman D. S., NeuroReport, 20, 150-156 (2009).

7) Sun F., Kanthasamy A., Anantharam V., Kanthasamy A. G., Pharmacol. Ther, 114, 327-344 (2007).

8) Davis G. C., Williams A. C., Markey S. P., Ebert M. H., Caine E. D., 
Reichert C. M., Kopin I. J., Psychiatry Res., 1, 249-254 (1979).

9) Langston J. W., Ballard P., Tetrud J. W., Irwin I., Science, 219, 979980 (1983).

10) Chiba K., Trevor A., Castagnoli N. Jr., Biochem. Biophys. Res. Commun., 120, 574-578 (1984).

11) Maguire-Ziss K. A., Pharmacol. Res., 58, 271-280 (2008).

12) Oksman M., Tanila H., Yavich L., Neuropharmacology, 56, 647-652 (2009).

13) Saito S., Kawabata J., Tetrahedron, 61, 8101-8108 (2005).

14) An L. J., Guan S., Shi G. F., Bao Y. M., Duan Y. L., Jiang B., Food Chem. Toxicol., 44, 436-443 (2006).
15) Mutoh T., Tokuda A., Marini A. M., Fujiki N., Brain Res., 661, 5155 (1994).

16) Nagata K., Takei N., Nakajima K., Saito H., Kohsaka S., J. Neurosci. Res., 34, 357-363 (1993).

17) Xu J., Wei C. Z., Xu C. Q., Bennett M. C., Zhang G. H., Li F. C., Tao E. N., Brain Res., 139, 220-225 (2007).

18) Liu G. W., Zhang C. Y., Yin J. J., Li X., Cheng F. R., Li Y. H., Yang H., Uéda K., Chan P., Yu S., Neurosci. Lett., 454, 187-192 (2009).

19) Banerjee R., Starkov A. A., Beal M. F., Thomas B., Biochim. Biophys. Acta, doi:10.1016/j.bbadis.2008.11.007 (2008). 